\section{P2-524 FOOD INSECURITY IN THE PARIS METROPOLITAN AREA. AN ANALYSIS OF THE SIRS COHORT IN 2010}

doi:10.1136/jech.2011.142976m.51

${ }^{1,2} \mathrm{~J}$ Martin-Fernandez, ${ }^{1,2} \mathrm{~F}$ Grillo, ${ }^{4} \mathrm{~F}$ Caillavet, ${ }^{1,3}{ }^{\mathrm{P}}$ Chauvin.* ${ }^{1}$ INSERM, U707, Research Team on the Social Determinants of Health and Healthcare, Paris, France; ${ }^{2}$ UPMC Univ Paris 06, UMR-S 707, Paris, France, ${ }^{3}$ AP-HP, Hôpital Saint Antoine, Unité de Santé publique, Paris, France; ${ }^{4}$ INRA-ALISS, UR 130, Ivry, France

Background Food insecurity exists whenever the availability of nutritionally adequate and safe foods or the ability to acquire acceptable foods in socially acceptable ways is limited or uncertain. It is a multidimensional, dynamic phenomenon that exists even in industrialised countries. The aim of this work was to estimate its prevalence and some of its social determinants in the Paris metropolitan area (PMA).

Methods We used data from the SIRS cohort, a longitudinal health and socio epidemiological, population based, and representative survey of the general population of the PMA. This cross sectional analysis was based on the 2010 data. Food insecurity was estimated using the US-HFSS and computed in a three categories variable: food secure, low food secure and very low food secure and in a dichotomous variable for logistic regressions (food insecurity: yes/no).

Results In 2010 about 94\% of the households living in Paris metropolitan area were food secure. The remaining households $-6.3 \%, 95 \% \mathrm{CI}=[3.9$ to 2.7$]$, that is, approximatively 500000 individuals - had experienced food insecurity during the past year. Almost $2.5 \%$ of the household living in Paris metropolitan area were in very low food security. If income was a major determinant of food insecurity (FI), some household's characteristics were associated with FI: single parent's families $(O R=2.79, p<0.001)$ and one person households $(\mathrm{OR}=3.95, \mathrm{p}<0.001)$. Households headed by employee had also more risks to be FI $(O R=2.0, p<0.025$, ref=executives).

Conclusion Household food insecurity is not rare in the PMA which needs further researches and a specific attention of social and welfare policies.

\section{P2-525 TREND OF THE SCHISTOSOMIASIS MANSONI IN MARANHÃO STATE: 1997 TO 2003}

doi:10.1136/jech.2011.142976m.52

S P D Cantanhede, I E Mattos, * A P Ferreira. Oswaldo Cruz Foundation, National School of Public Health, Rio de Janeiro, Rio de Janeiro, Brazil

Introduction Schistosomiasis is still a major public health problem in Brazil, where it is assumed that there 2500000 to 8000000 cases of the disease, despite the development of control activities. The North-eastern region, where is situated the state of Maranhão, is the most affected area. This study analyses trends of the percentage of positive cases of Schistosomiasis in Maranhao, between 1997 and 2003.

Methods We analysed a time series of percentages of positive cases, grouped according to 15 Regional Health Authorities in the state, available in the information system of the Schistosomiasis Control Program (SISPCE). Trend analysis was performed through polynomial regression models. Statistical significance, coefficients of determination $\left(R^{2}\right)$ and residuals were used to define the best model. Results The percentage of positive cases of schistosomiasis amounted to $7.42 \%$ in the study period. Two Regional Health Authorities showed constant downward trends with an average annual percentage of positive cases of 1.72 and 0.66 , respectively. Meanwhile, the others showed upward trends.

Conclusions The declining trends suggest the possibility of underreporting. It is believed that data registration in the Regional Health
Authorities has been affected by changes in the activities of the SISPCE, due to decentralisation of the Program to municipalities. In some cases, best living conditions could have contributed to the observed declines. However, deficit of environmental sanitation and poverty are significant in the majority of the state and certainly are related to the increments observed in the other regionals.

\section{P2-526 SOCIAL DETERMINANTS OF DEPRESSIVE SYMPTOMS AMONG OLDER ADULTS IN LOW- AND MIDDLE-INCOME COUNTRIES}

doi:10.1136/jech.2011.142976m.53

B McKinnon, ${ }^{*}$ S Harper. McGill University, Montreal, Quebec, Canada

Background Consistent evidence has linked depression to social factors among older people in rich countries; however, little is known for low- or middle-income countries (LMIC) and virtually no research exists that is comparable across LMIC. Understanding the relationship between key socioeconomic factors and depressive symptoms among older people in resource-poor contexts is essential for developing mental health policies.

Methods To investigate social determinants of depressive symptoms among older adults in LMIC, we conducted a cross-sectional analysis of adults age $50+$ from 51 countries that participated in the World Health Survey in 2002-2003. Using multivariable ordinal logistic regression models, we examined the association between socioeconomic predictors and the severity of depressive symptoms. Results Similar to patterns from rich countries, more severe depressive symptoms were reported among older, female, less-educated, poorer, and urban-dwelling individuals. Living arrangement also emerged as an important predictor that exhibited substantial heterogeneity across countries. In Southeast Asian countries, the odds of reporting more severe depressive symptoms was 2.6 (95\% CI 1.5 to 4.7) times higher for individuals living alone compared to in intergenerational households. In African countries, individuals living in skipped-generation households (only older people and dependent children) reported significantly worse symptoms. Further analyses will incorporate country-level predictors (eg, availability of pensions, HIV/AIDS mortality) to explain some between-country variation.

Conclusions In addition to established socioeconomic determinants, living alone or in skipped generation households is associated with an increased risk of depressive symptoms among older people.

\section{P2-527 PREVALENCE OF DEPRESSION IN THE BRAZILIAN FAMILY HEALTHCARE STRATEGY: A CROSS-SECTIONAL STUDY IN SÃO PAULO AND MANAUS}

doi:10.1136/jech.2011.142976m.54

${ }^{1} \mathrm{P}$ R Menezes, ${ }^{*}{ }^{1} \mathrm{M}$ Scazufca, ${ }^{2} 0$ P Almeida, ${ }^{3} \mathrm{R}$ Araya, ${ }^{4} \mathrm{M}$ M Cruz, ${ }^{5} \mathrm{~L}$ A D'Angelo, ${ }^{1,5} \mathrm{~L}$ Garcia, ${ }^{1} \mathrm{M}$ Mogadouro, ${ }^{5} \mathrm{C}$ Santana, ${ }^{1} \mathrm{R} N$ Santos, ${ }^{1} \mathrm{~S}$ C Sawada, ${ }^{1} \mathrm{C}$ Seabra, ${ }^{1} S$ A Silva, ${ }^{1}$ A T C Silva, ${ }^{4}$ A M M Souza, ${ }^{4}$ M Trindade, ${ }^{4} E$ E Ribeiro. ${ }^{1}$ University of Sao Paulo, Sao Paulo, Brazil; ' 2 University of Western Australia, Perth, Australia; ${ }^{3}$ University of Bristol, Bristol, UK; ${ }^{4}$ Amazon State University, Manaus, Brazil; ${ }^{5}$ Associação Saúde da Família, Sao Paulo, Brazil

Introduction The Family Healthcare Strategy (FHCS), the new model of Primary Care adopted by the Brazilian Ministry of Health, with its territoriality and regular visits by community health workers, provides an excellent network for treating depressed elderly. We aimed to estimate the prevalence of depression among older adults registered with FHCS teams in two large Brazilian cities, one in the Southeast and the other in the Amazon.

Method Survey with adults aged 60 and over registered in FHCS teams' lists in São Paulo and Manaus. Participants were randomly drawn from lists of clients of 23 teams in São Paulo and 29 in Manaus, and were interviewed at home. Depression was assessed 
with the PHO-9, and classified as mild or moderate to severe. Ordinal logistic regression was used to assess statistical associations between depression and sex, age, illiteracy, family income and centre.

Results 1020 participants were included, 446 in São Paulo and 574 in Manaus, of whom 248 (24.3\%; 95\% CI 21.7 to 27.1$)$ were classified as mild depression and 93 (9.1\%; $95 \%$ CI 7.4 to 11.1$)$ as moderate to severe depression. The prevalence of mild and moderate to severe depression was very similar in the two centres. Women were almost twice as likely as men to present with depression of any severity. We did not find any association between depression and age, illiteracy or family income.

Conclusion Depression is highly prevalent among primary care clients, especially women. Mental healthcare must be integrated into primary care in order to reduce the treatment gap for depression.

\section{P2-528 ADULT PERCEPTIONS OF YOUTH MENTAL HEALTH ISSUES IN A CANADIAN PROVINCE}

doi:10.1136/jech.2011.142976m.55

${ }^{1}$ A Metcalfe, ${ }^{*}{ }^{1,2} \mathrm{~S}$ Tough, ${ }^{2} \mathrm{~J}$ Salegio, ${ }^{2} \mathrm{~T}$ Hanson. ${ }^{1}$ University of Calgary, Calgary, Alberta, Canada; ${ }^{2}$ Alberta Centre for Child, Family \& Community Research, Edmonton, Alberta, Canada

Although $15 \%$ of Canadian youth experience mental health problems, barriers to disclosure and treatment exist. This population-based study assessed adult's beliefs about the prevalence of mental illness among youth, treatment for mental illness, and comfort interacting with youth with moderate mental health problems. In 2010 a random sample of 1203 adults residing in Alberta Canada were surveyed. $\chi^{2}$ Tests and t-tests were used to understand responses by demographic factors. Logistic regression was used to determine factors predictive of Albertans comfort in interacting with youth with moderate mental health problems. Twenty percent were able to correctly identify the prevalence of youth mental health problems. Over $50 \%$ stated that they believed that $<10 \%$ of youth with mental health problems received treatment. Approximately $70 \%$ of the sample reported they would be comfortable interacting with youth with moderate mental health problems in work, school, social and community settings. Consistent predictors of comfort interacting with youth with moderate mental health problems included: being between the ages of 18-24, high school completion, Caucasian ethnicity, and annual household income $>\$ 40000 /$ year. There are meaningful gaps in Albertans understanding of the prevalence of youth mental health issues, but the majority of adults would be comfortable interacting with youth with moderate mental health problems. Many respondents identified that youth with mental health problems may not be receiving treatment. Increased public awareness about the prevalence and detrimental impact of youth mental health issues may help policy makers allocate resources to effective screening and treatment for youth with mental health concerns.

\section{P2-529 CHILDREN LIVING IN POOR HYGIENE AND SANITATION CONDITIONS: WHY DOES IT MATTER?}

doi:10.1136/jech.2011.142976m.56

'S Nadeem,* ${ }^{2} \mathrm{~B}$ I Avan. 'Aga Khan University-Human Development Programme, Karachi, Pakistan; ${ }^{2}$ Immpact, School of Medicine \& Dentistry, University of Aberdeen, Aberdeen, UK

Background It is estimated that at least 200 million children, mostly from developing countries, suffer from developmental delays. Poor hygiene and sanitation conditions are known to influence growth faltering and increased risk of morbidity and mortality, yet little is know about role of these conditions on child growth and development. The study aims to contribute to an understanding of the relationship between family hygiene, sanitary conditions and psychomotor development in the first 3 years of life.
Method A cross sectional household survey was conducted in urban and rural Sindh, Pakistan, by trained personnel. Children aged $<3$ $(n=1244)$ were assessed at home visits using (1) Bayley's Infant Developmental Scale for psychomotor development; (2) anthropometric status, and (3) socio-economic, hygiene and sanitation conditions via maternal interview. Socioeconomic, hygiene and sanitation indices are created using principal component analyses

Results Rural areas are highly correlated with hygiene index $(\mathrm{HI})$ and sanitation index (SI), as compared to their urban counterparts. Age and sex adjusted SI is highly correlated with socioeconomic index (SEI). Multilevel modelling analysis showed that SI is significantly associated with child's delayed development, stunting and being underweight; while $\mathrm{HI}$ is associated with underweight and stunting status of children, independent of SEI and rural-urban neighbourhood.

Conclusion Due to a strong association between hygiene and sanitation conditions and development, recognition should be given to the growth and developmental needs of children living in such conditions.

\section{P2-530 SOCIODEMOGRAPHIC PROFILE AND EFFICACY OF 4 ART REGIMES IN HIV PATIENTS REGISTERED AT AN ART CENTER-A DEVELOPING NATION SCENARIO}

doi:10.1136/jech.2011.142976m.57

V Nagaraja,* M A Khan, G Bhat. MMC \& Rl, Karnataka, India

Introduction People living with HIV in 2008 worldwide are 33.4 million with 2.7 million new cases and 2 million deaths. Southeast Asia has 4.7 million HIV patients. Five countries account for majority of the HIV infections-India (2.31 million), Thailand, Myanmar, Indonesia and Nepal. Hence we sought to study the sociodemographic profile of HIV patients registered at ART center in Mysore. Cluster Designation 4 (CD4) count at the initiation of ART and 6 months thereafter were compared.

Methods The study sample was taken from the records of the ART center at Krishnarajendra hospital, Mysore that consisted of 1702 patients on ART from June 2007 to March 2010. Socioeconomic $\&$ demographic details, sexual practices, HIV status (WHO staging) \& CD4 counts at the initiation and after 6 months of treatment were collected.

Results Majority of the patients on ART (77\%) were males between 21 and 40 years, educated heterosexuals who mainly presented with fever and weight loss. $68 \%$ of spouses knew their HIV status and among them $36 \%$ were HIV positive. Majority of them were categorised under stage 3 . There was an improvement in mean CD4 count by around 200 . ZDV +LMV +NVP regime showed a significant increase in mean CD4count over the other three regimes and there was statistically significant correlation.

Conclusion Most of the HIV patients were young educated heterosexual males and showed a significant increase in the CD4 cell count after 6 months of antiretroviral therapy.

\section{P2-531 A COMPARATIVE STUDY OF FACTORS AFFECTING PSYCHOLOGICAL WELL-BEING OF URBAN AND RURAL ADOLESCENTS}

doi:10.1136/jech.2011.142976m.58

V Nagaraja, ${ }^{*}$ M A Khan, G Bhat. Mysore Medical College and Research Institute, Mysore, Karnataka, India

Aims and Objectives We have limited studies and data allowing us a bleak tunnel vision into the world of adolescent mental health. A comparative study was initiated to have a better understanding of 